\title{
First Principles Investigation of the Structural and Electrochemical Properties of $\mathrm{Na}_{4} \mathrm{P}_{2} \mathrm{~S}_{6}$ and $\mathrm{Li}_{4} \mathrm{P}_{2} \mathrm{~S}_{6}$.
}

\author{
Larry E. Rush Jr. ${ }^{\text {, N N. A. W. Holzwarth }}{ }^{\mathrm{a}}$ \\ ${ }^{a}$ Department of Physics, Wake Forest University, Winston-Salem, NC 27109-5709 USA
}

\begin{abstract}
First principles simulations are used to examine the structural and physical properties of $\mathrm{Na}_{4} \mathrm{P}_{2} \mathrm{~S}_{6}$ in comparison with its $\mathrm{Li}_{4} \mathrm{P}_{2} \mathrm{~S}_{6}$ analog. Four model structures are considered including the $C 2 / m$ structure recently reported by Kuhn and coworkers from their analysis of single crystals of $\mathrm{Na}_{4} \mathrm{P}_{2} \mathrm{~S}_{6}$, and three structures related to the $P 6_{3} / m c m$ structure with $\mathrm{P}$ site disorder found in 1982 by Mercier and co-workers from their analysis of single crystals of $\mathrm{Li}_{4} \mathrm{P}_{2} \mathrm{~S}_{6}$. The computational results indicate that both $\mathrm{Na}_{4} \mathrm{P}_{2} \mathrm{~S}_{6}$ and $\mathrm{Li}_{4} \mathrm{P}_{2} \mathrm{~S}_{6}$ have the same disordered ground state structures consistent with the $P 6_{3} / \mathrm{mcm}$ space group, while the optimized $C 2 / \mathrm{m}$ structures have higher energies by $0.1 \mathrm{eV}$ and $0.4 \mathrm{eV}$ per formula unit for $\mathrm{Na}_{4} \mathrm{P}_{2} \mathrm{~S}_{6}$ and $\mathrm{Li}_{4} \mathrm{P}_{2} \mathrm{~S}_{6}$, respectively. In modeling ionic conductivity in these materials, activation energies for $\mathrm{Na}$ ion vacancy migration were computed to be smaller than the $\mathrm{Li}$ analogs in all of the structural models. Interestingly, the results also indicate that if $\mathrm{Li}_{4} \mathrm{P}_{2} \mathrm{~S}_{6}$ could be prepared in the meta-stable $C 2 / m$ structure, the $\mathrm{Li}$ ion vacancy migration would have very small barriers along certain channels. Simulations of $\mathrm{Na}_{4} \mathrm{P}_{2} \mathrm{~S}_{6}(C 2 / m) / \mathrm{Na}$ interfaces indicate that they may be slightly less reactive than $\mathrm{Li}_{4} \mathrm{P}_{2} \mathrm{~S}_{6}\left(P 6_{3} / \mathrm{mcm}\right) / \mathrm{Li}$ interfaces.
\end{abstract}

\section{Introduction}

In the development of solid state battery technology, there has been some recent progress in finding promising $\mathrm{Na}$ ion conducting materials.[1] Kuhn and co-workers[2] recently reported an interesting new structure for $\mathrm{Na}_{4} \mathrm{P}_{2} \mathrm{~S}_{6}$. Motivated by possibilities for $\mathrm{Na}$ ion electrolytes in advance of experimental measurements, we computationally examine the structural and physical properties of $\mathrm{Na}_{4} \mathrm{P}_{2} \mathrm{~S}_{6}$ in comparison with $\mathrm{Li}_{4} \mathrm{P}_{2} \mathrm{~S}_{6}$. [3, 4] In particular, computations can be used to model the apparent structural differences between the two materials. $\mathrm{Li}_{4} \mathrm{P}_{2} \mathrm{~S}_{6}$ is known to have an interesting disordered structure which differs from Kuhn structure. Comparisons of the simulated $\mathrm{Na}$ and $\mathrm{Li}$ ion vacancy migration mechanisms for the various model structures can be used to predict ionic conductivity properties of the materials. Idealized interfaces between $\mathrm{Na}_{4} \mathrm{P}_{2} \mathrm{~S}_{6} / \mathrm{Na}$ are also investigated in order to predict electrolyte/anode interface stability.

This paper is organized as follows. Section 2 describes the computational methods used in this study. Section 3 presents the results for the energy and structural analysis in Sec. 3.1, for ion migration simulations in Sec. 3.2, and for interface simulations in Sec. 3.3. Discussions and conclusions are given in Sec. 4.

\section{Methods}

The calculational methods used in this work were the same as those used in previous studies of similar materials. [5,
4] Specifically, the calculations were based on density functional theory, $[6,7]$ using the projector augmented wave (PAW) $[8,9]$ formalism. The PAW basis and projector functions were generated by the ATOMPAW[10] code and used in both the ABINIT[11] and QUANTUM ESPRESSO[12] packages. The exchange-correlation functional was the local density approximation (LDA),[13] which has been shown to give excellent results for similar materials provided that a systematic $2 \%$ underestimate of the lattice size is taken into account for each of the 3 dimensions. [4] The partial densities of states were calculated as described in previous work, $[5,4]$ using weighting factors based on the charge within the augmentation spheres of each atom with radii $r_{c}^{\mathrm{Na}}=1.7, r_{c}^{\mathrm{Li}}=1.6, r_{c}^{\mathrm{P}}=1.7$, and $r_{c}^{\mathrm{S}}=1.7 \mathrm{in}$ bohr units.

The electronic structure calculations were performed with plane wave expansions of the wavefunctions including $|\mathbf{k}+\mathbf{G}|^{2} \leq 64$ bohr $^{-2}$ and with a Brillouin zone sampling grid density of at least $0.003 \mathrm{bohr}^{-3} / \mathbf{k}$-point. Structural parameters of the model systems, including the lattice constants and fractional atomic coordinates, were determined by optimizing the calculated total energy of each structure. The software program FINDSYM[14] was used to help analyze the symmetry properties of the optimized structures.

Vacancy, interstitial, and vacancy-interstitial pair defects were modeled using supercells consisting of 8 formula units. The simulations were performed on neutral supercells, adding a compensating uniform charge when modeling charged defects. The "nudged elastic band" (NEB) method, $[15,16,17]$ as included in the QUANTUM ESPRESSO package was used to estimate the $\mathrm{Li}^{+} / \mathrm{Na}^{+}$migration en- 
ergies, $E_{m}$, in supercell models. For this analysis it was assumed that 5 images between each meta-stable configuration was sufficient to estimate the path energies. The migration energies determined from the NEB calculations can be related to the experimental conductivity, $\sigma$, and to measurements as a function of temperature, $T$, through the Arrhenius relationship

$$
\sigma=\frac{C}{T} \mathrm{e}^{-E_{A} / k T},
$$

where $C$ denotes a temperature independent constant for the sample, $k$ denotes the Boltzmann constant, and $E_{A}$ represents the activation energy for $\mathrm{Na} / \mathrm{Li}$ ion migration. For nearly perfect crystals, thermal processes must initiate the formation of a vacancy and interstitial pair with energy $E_{f}$, so that the activation energy is related to the migration energy, $E_{m}$, according to $E_{A}=E_{m}+\frac{1}{2} E_{f}$. For crystals with a significant population of native defects, we expect $E_{A}=E_{m} \cdot[18]$

Visualizations were constructed using the $X C r y S D E N[19$, 20], VESTA[21] and CrystalMaker[22] software packages.

\section{Results}

\subsection{Structural properties}

Mercier's structural analysis[3] for $\mathrm{Li}_{4} \mathrm{P}_{2} \mathrm{~S}_{6}$ named the space group $P 6_{3} / \mathrm{mcm}$ (\# 193) in the International Table of Crystallography)[23] and designated the $\mathrm{P}$ sites with Wyckoff label $4 e$ and site symmetry $3 . m$ as having fractional occupancy 0.5. We have recently re-examined $\mathrm{Li}_{4} \mathrm{P}_{2} \mathrm{~S}_{6}$ experimentally and computationally finding that the disorder can be explained in terms of energetic degeneracy in the placement of the $\mathrm{P}_{2} \mathrm{~S}_{6}$ building blocks within the structure. [4] The structure for $\mathrm{Na}_{4} \mathrm{P}_{2} \mathrm{~S}_{6}$ found by Kuhn and co-workers[2] appears to be entirely different from the Mercier structure; however, upon closer examination, we find that it is possible to find a geometric relationship between all of the structures. In fact, as discussed below, our calculations indicate that the Kuhn structure is not the lowest energy structure of $\mathrm{Na}_{4} \mathrm{P}_{2} \mathrm{~S}_{6}$.

The four structural forms for both $\mathrm{Na}_{4} \mathrm{P}_{2} \mathrm{~S}_{6}$ and $\mathrm{Li}_{4} \mathrm{P}_{2} \mathrm{~S}_{6}$ that comprise the current study are summarized in Table 1 in terms of their optimized lattice parameters. The unique fractional coordinates of $\mathrm{Na}_{4} \mathrm{P}_{2} \mathrm{~S}_{6}$ and $\mathrm{Li}_{4} \mathrm{P}_{2} \mathrm{~S}_{6}$ in the four crystal structures are listed in Table 2 . The four structures are labeled according to their space groups $-C 2 / m$, corresponding to the Kuhn structure [2], and $P \overline{3} 1 \mathrm{~m}$, Pnnm, and Pnma, corresponding to the structures labeled Struc. (b), (c), and (d) studied in Ref. [4]. $P \overline{3} 1 \mathrm{~m}$ corresponds to a subgroup of the Mercier space group. The other two structures, which happen to have the space groups Pnnm and Pnma, are constructed from larger supercells and are examples of the disordered Mercier structure [3].

The results in Table 1 show that the ratio of the measured and computed lattice constants for $\mathrm{Na}_{4} \mathrm{P}_{2} \mathrm{~S}_{6}$ in the $C 2 / m$ structure follows the expected 1.02 scaling very well
Table 1: Lattice parameters for the four model structures of $\mathrm{Na}_{4} \mathrm{P}_{2} \mathrm{~S}_{6}$ and $\mathrm{Li}_{4} \mathrm{P}_{2} \mathrm{~S}_{6}$. For each structure, the table lists the space group labels and ID numbers[23] as well as $Z$, the number of formula units per unit cell. The results are given for the calculated lattice parameters $(a, b, c, \beta)$, in $\AA$ and degree units, respectively, for the optimized structures. Corresponding experimental lattice parameters are listed in parentheses when available - Ref. [2] given in the $C 2 / m$ section and Ref. [4] given in the Pnnm section.

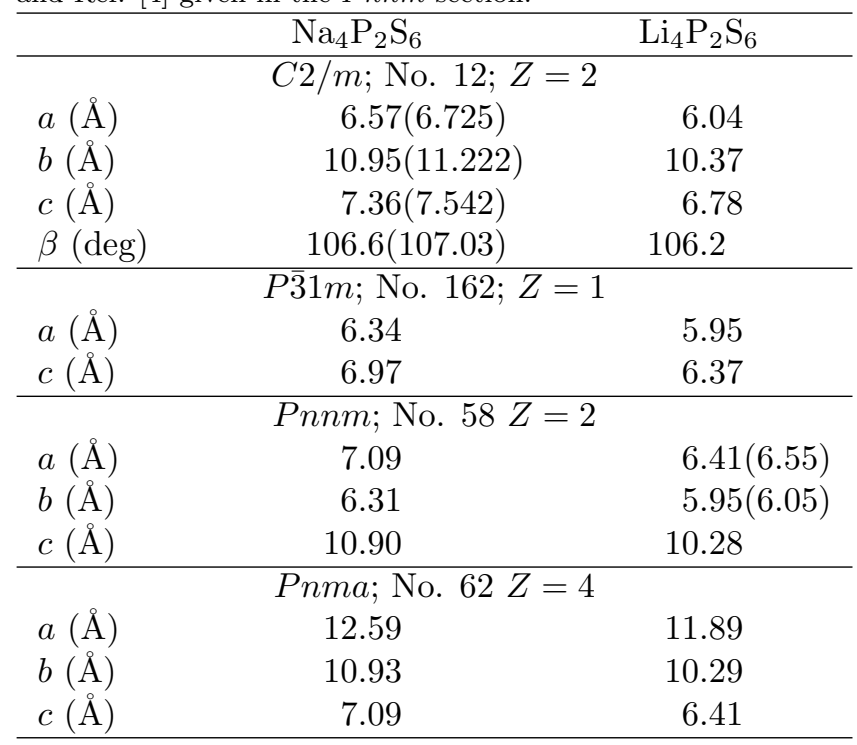

based on the experimental results of Ref. [2]. Similarly, the computed lattice constants for $\mathrm{Li}_{4} \mathrm{P}_{2} \mathrm{~S}_{6}$, as approximated by the Pnnm and Pnma structures, also follow the expected 1.02 scaling based on the low temperature measurements of Ref. [4]. The computed fractional coordinates of $\mathrm{Na}_{4} \mathrm{P}_{2} \mathrm{~S}_{6}$ in the $C 2 / m$ structure agree very well with the experimental results of Ref. [2].

The four structures considered in this study are geometrically related. The $P \overline{3} 1 \mathrm{~m}$ structure (labeled as Struc. (b) in Ref. [4]) is based on a hexagonal bravais lattice shown in Fig. 1. The lattice parameters $a=b$ and $c$ for $\mathrm{Na}_{4} \mathrm{P}_{2} \mathrm{~S}_{6}$ and $\mathrm{Li}_{4} \mathrm{P}_{2} \mathrm{~S}_{6}$ in this structure are given in Table 1. As will be shown below, the Kuhn[2] $C 2 / m$ structure can be derived from a orthorhombic supercell of the $P \overline{3} 1 \mathrm{~m}$ structure. The Pnnm and Pnma structures (labeled as Struc. (c) and (d) in Ref. [4]) represent 2 of many possible realizations of the disordered $\mathrm{P}_{3} / \mathrm{mcm}$ structure of $\mathrm{Li}_{4} \mathrm{P}_{2} \mathrm{~S}_{6}$ analyzed by Mercier[3] based on orthorhombic supercells of the basic hexagonal structure.

The $P \overline{3} 1 \mathrm{~m}$ structure shown in Fig. 1 has two inequivalent $\mathrm{Na}$ positions indicated by the two shades of blue in the diagram. In the view projected along the $c$-axis (Fig. 1a) the two Na sites are superposed, while the perpendicular view (Fig. 1b) shows that the $d$ site Na's are located in a plane between the $\mathrm{P}_{2} \mathrm{~S}_{6}$ units, while the $c$ site $\mathrm{Na}$ 's are located in a plane which also contains the $\mathrm{P}_{2} \mathrm{~S}_{6}$ units.

The Kuhn structure[2] of $\mathrm{Na}_{4} \mathrm{P}_{2} \mathrm{~S}_{6}$ is shown in Fig. 2 in its conventional setting. Its relationship to the hexagonal vectors defined in Fig. 1 is as follows:

$$
\mathbf{a}_{K u h n}=\mathbf{a}_{h e x}, \quad \mathbf{b}_{K u h n}=\mathbf{o}_{h e x}, \quad \mathbf{c}_{K u h n} \rightarrow \mathbf{c}_{h e x} .
$$


Table 2: Computed unique fractional coordinates for $\mathrm{Na}_{4} \mathrm{P}_{2} \mathrm{~S}_{6}$ and $\mathrm{Li}_{4} \mathrm{P}_{2} \mathrm{~S}_{6}$ in the four crystal structures. For comparison, fractional coordinates for $\mathrm{Na}_{4} \mathrm{P}_{2} \mathrm{~S}_{6}$ in the $C 2 / m$ structure reported by Ref. [2] is also given as indicated with "*". The site multiplicity and label is listed in the second column in terms of the Wyckoff notation. [23]

\begin{tabular}{|c|c|c|c|}
\hline Atom & Site & $\mathrm{Na}_{4} \mathrm{P}_{2} \mathrm{~S}_{6}$ & $\mathrm{Li}_{4} \mathrm{P}_{2} \mathrm{~S}_{6}$ \\
\hline \multicolumn{4}{|c|}{$C 2 / m ;$ No. 12} \\
\hline \multirow[t]{2}{*}{$\mathrm{Na} / \mathrm{Li}$} & $4 g$ & $(0,0.339,0)$ & $(0,0.333,0)$ \\
\hline & & $(0, .337,0)^{*}$ & \\
\hline \multirow[t]{2}{*}{$\mathrm{Na} / \mathrm{Li}$} & $4 h$ & $\left(0,0.186, \frac{1}{2}\right)$ & $\left(0,0.178, \frac{1}{2}\right)$ \\
\hline & & $\left(0,0.185, \frac{1}{2}\right)^{*}$ & \\
\hline \multirow[t]{2}{*}{$\mathrm{P}$} & $4 i$ & $(0.054,0,0.160)$ & $(0.056,0,0.169)$ \\
\hline & & $(0.053,0,0.156)^{*}$ & \\
\hline \multirow[t]{2}{*}{ S } & $4 i$ & $(0.789,0,0.247)$ & $(0.765,0,0.260)$ \\
\hline & & $(0.794,0,0.241)^{*}$ & \\
\hline \multirow[t]{3}{*}{ S } & $8 j$ & $(0.726,0.346,0.232)$ & $(0.738,0.335,0.240)$ \\
\hline & & $(0.723,0.350,0.231)^{*}$ & \\
\hline & \multicolumn{3}{|c|}{$P \overline{3} 1 m ;$ No. 162} \\
\hline $\mathrm{Na} / \mathrm{Li}$ & $2 c$ & $\left(\frac{1}{3}, \frac{2}{3}, 0\right)$ & $\left(\frac{1}{3}, \frac{2}{3}, 0\right)$ \\
\hline $\mathrm{Na} / \mathrm{Li}$ & $2 d$ & $\left(\frac{1}{3}, \frac{2}{3}, \frac{1}{2}\right)$ & $\left(\frac{1}{3}, \frac{2}{3}, \frac{1}{2}\right)$ \\
\hline $\mathrm{P}$ & $2 e$ & $(0,0,0.165)$ & $(0,0,0.176)$ \\
\hline $\mathrm{S}$ & $6 k$ & $(0.308,0,0.240)$ & $(0.331,0,0.248)$ \\
\hline \multicolumn{4}{|c|}{ Pnnm; No. 58} \\
\hline $\mathrm{Na} / \mathrm{Li}$ & $4 e$ & $(0,0,0.341)$ & $(0,0,0.334)$ \\
\hline $\mathrm{Na} / \mathrm{Li}$ & $4 f$ & $\left(0, \frac{1}{2}, 0.824\right)$ & $\left(0, \frac{1}{2}, 0.832\right)$ \\
\hline $\mathrm{P}$ & $4 g$ & $(0.161,0.000,0)$ & $(0.174,0.000,0)$ \\
\hline S & $8 h$ & $(0.235,0.845,0.155)$ & $(0.245,0.834,0.166)$ \\
\hline S & $4 g$ & $(0.232,0.310,0)$ & $(0.246,0.331,0)$ \\
\hline \multicolumn{4}{|c|}{ Pnma; No. 62} \\
\hline $\mathrm{Na} / \mathrm{Li}$ & $8 d$ & $(0.632,0.589,0.000)$ & $(0.626,0.584,0.000)$ \\
\hline $\mathrm{Na} / \mathrm{Li}$ & $8 d$ & $(0.119,0.579,0.000)$ & $(0.125,0.583,0.000)$ \\
\hline $\mathrm{P}$ & $4 c$ & $\left(0.625, \frac{1}{4}, 0.160\right)$ & $\left(0.625, \frac{1}{4}, 0.176\right)$ \\
\hline $\mathrm{P}$ & $4 c$ & $\left(0.625, \frac{1}{4}, 0.839\right)$ & $\left(0.625, \frac{1}{4}, 0.829\right)$ \\
\hline S & $8 d$ & $(0.297,0.595,0.235)$ & $(0.292,0.584,0.244)$ \\
\hline S & $8 d$ & $(0.452,-0.095,0.769)$ & $(0.458,-0.084,0.755)$ \\
\hline S & $4 c$ & $\left(0.471, \frac{1}{4}, 0.760\right)$ & $\left(0.459, \frac{1}{4}, 0.755\right)$ \\
\hline S & $4 c$ & $\left(0.781, \frac{1}{4}, 0.230\right)$ & $\left(0.791, \frac{1}{4}, 0.245\right)$ \\
\hline
\end{tabular}

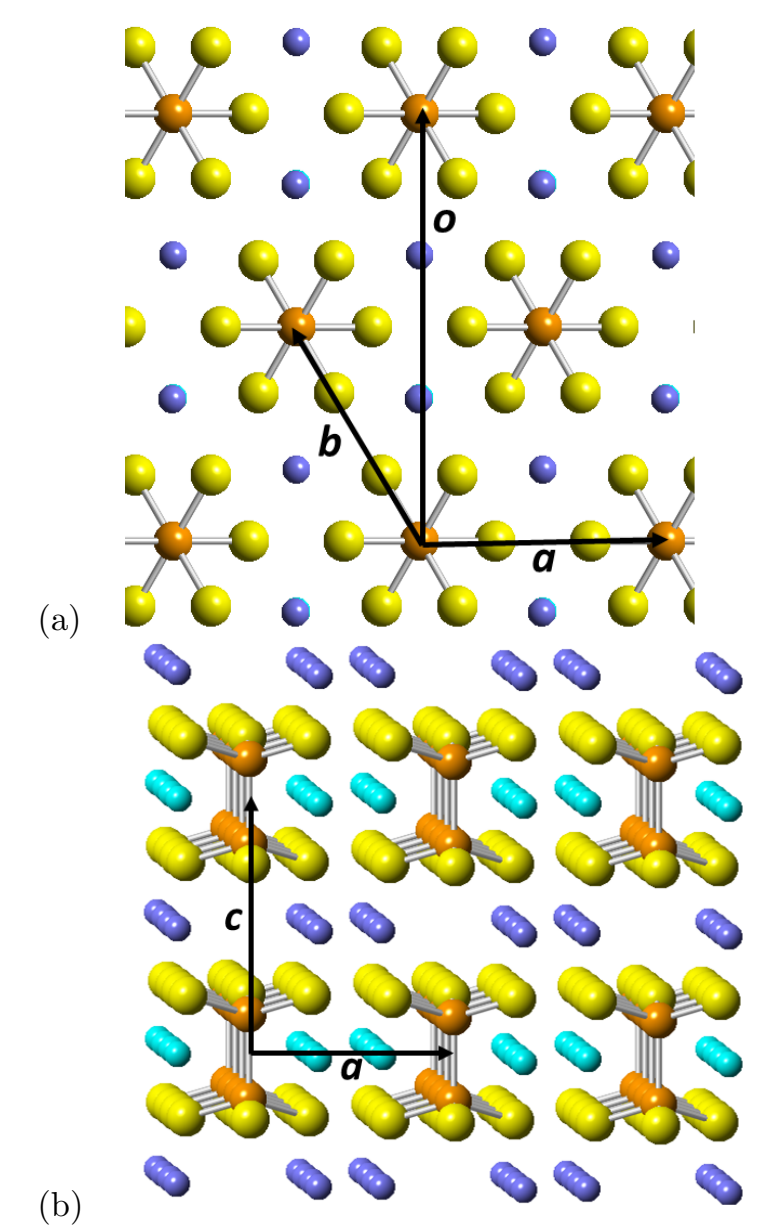

Figure 1: (Color online.) Ball and stick model of $\mathrm{Na}_{4} \mathrm{P}_{2} \mathrm{~S}_{6}$ in the $P \overline{3} 1 \mathrm{~m}$ structure showing (a) a projection onto the hexagonal plane and (b) a view including the $c$-axis. The $\mathrm{Na}, \mathrm{P}$, and $\mathrm{S}$ sites are represented by blue, orange, and yellow balls, respectively. The two shades of blue indicate the inequivalent $\mathrm{Na}$ sites. The vectors $\mathbf{a}, \mathbf{b}$, and $\mathbf{c}$ represent the conventional hexagonal lattice vectors, while $\mathbf{o}$ shows a corresponding orthorhombic lattice vector. 
(a)
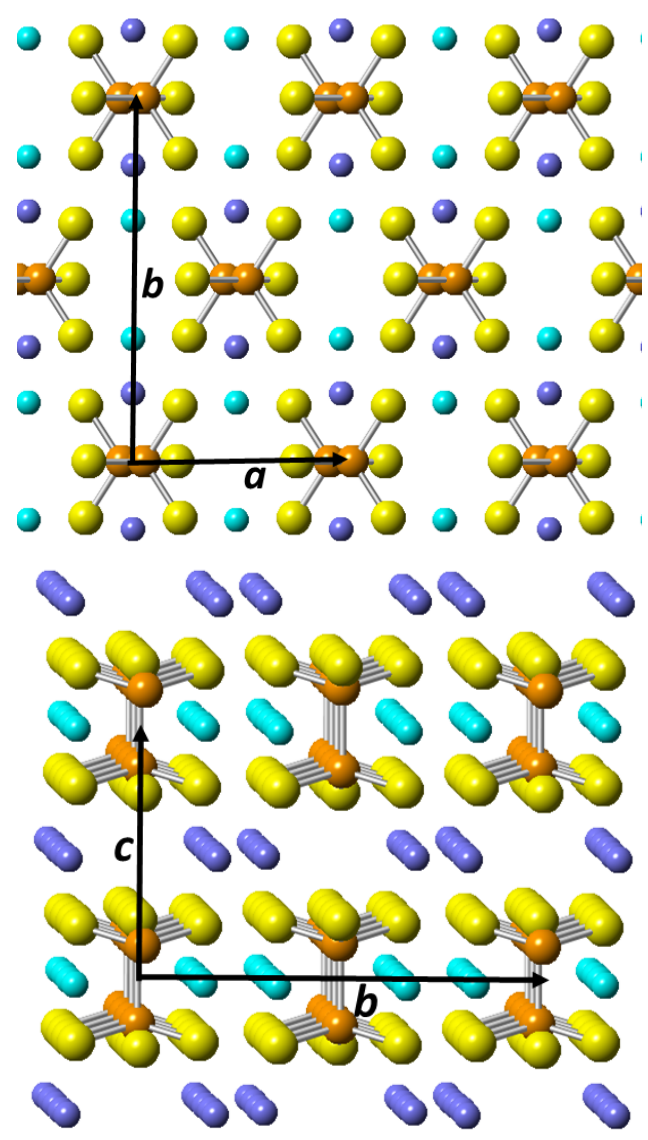

(b)

Figure 2: (Color online.) Ball and stick model of $\mathrm{Na}_{4} \mathrm{P}_{2} \mathrm{~S}_{6}$ in the conventional $C 2 / m$ structure using the same ball convention as in in Fig. 1.

While from the viewpoint of Fig. 2b, the structure looks very similar to Fig. 1, the $\mathbf{a}_{K u h n}$ and $\mathbf{c}_{K u h n}$ axes are no longer orthogonal. Figure 2a shows the structure as projected in $\mathbf{a}_{K u h n}-\mathbf{b}_{K u h n}$ planes. Here it is evident that $\mathrm{Na}$ sites within a given $\mathbf{a}_{K u h n}-\mathbf{b}_{K u h n}$ plane are arranged in an approximately hexagonal pattern. The approximately hexagonal patterns of the sites with Wyckoff labels $g$ and $h$ are shifted with respect to each other in this projection unlike the superposed $c$ and $d$ Na sites found in the $P \overline{3} 1 \mathrm{~m}$ structure.

The other structures in this study, which happen to have the space group symmetries Pnnm and Pnma, were derived from analyzing the disorder structures of $\mathrm{Li}_{4} \mathrm{P}_{2} \mathrm{~S}_{6}$ in terms of the placements of the $\mathrm{P}_{2} \mathrm{~S}_{6}$ units along the hexagonal $c$-axis. The Pnnm structure shown in Fig. 3 in its conventional setting, has the lattice relationships to the hexagonal vectors defined in Fig. 1 as follows:

$$
\mathbf{a}_{\text {Pnnm }}=\mathbf{c}_{\text {hex }}, \quad \mathbf{b}_{\text {Pnnm }}=\mathbf{a}_{\text {hex }}, \quad \mathbf{c}_{\text {Pnnm }}=\mathbf{o}_{\text {hex }} .
$$

The Pnma structure shown in Fig. 4 in its conventional setting, has the lattice relationships to the hexagonal vectors defined in Fig. 1 as follows:

$$
\mathbf{a}_{P n m a}=2 \mathbf{a}_{h e x}, \quad \mathbf{b}_{P n m a}=\mathbf{o}_{h e x}, \quad \mathbf{c}_{P n m a}=\mathbf{c}_{h e x} .
$$

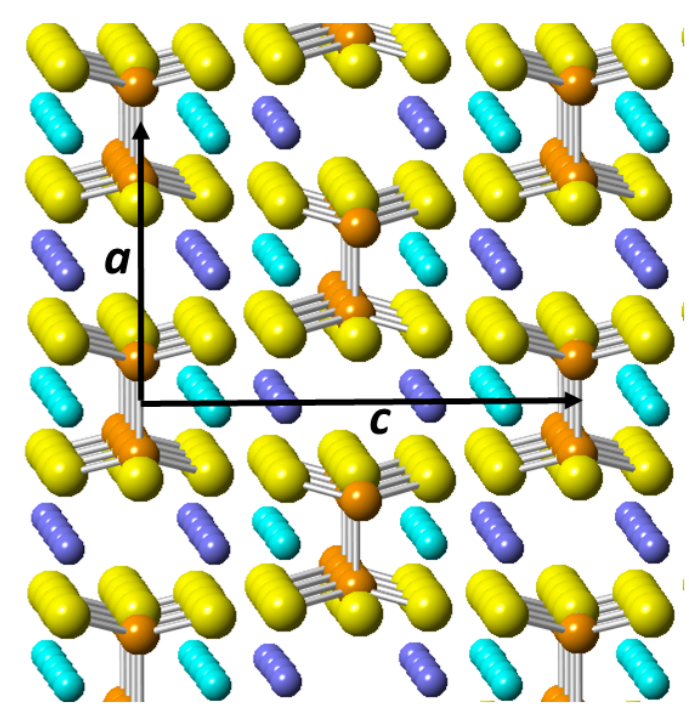

Figure 3: (Color online.) Ball and stick model of $\mathrm{Na}_{4} \mathrm{P}_{2} \mathrm{~S}_{6}$ in the conventional Pnnm structure using the same ball convention as in in Fig. 1.

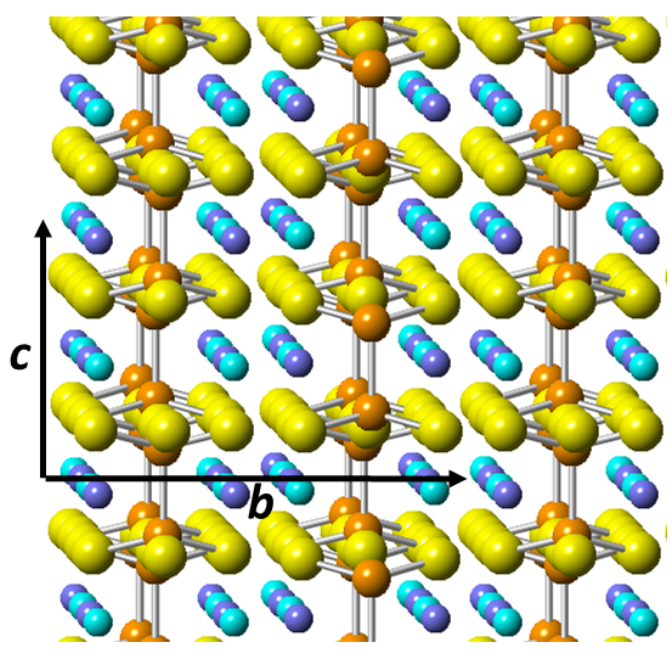

Figure 4: (Color online.) Ball and stick model of $\mathrm{Na}_{4} \mathrm{P}_{2} \mathrm{~S}_{6}$ in the conventional Pnma structure using the same ball convention as in in Fig. 1. 


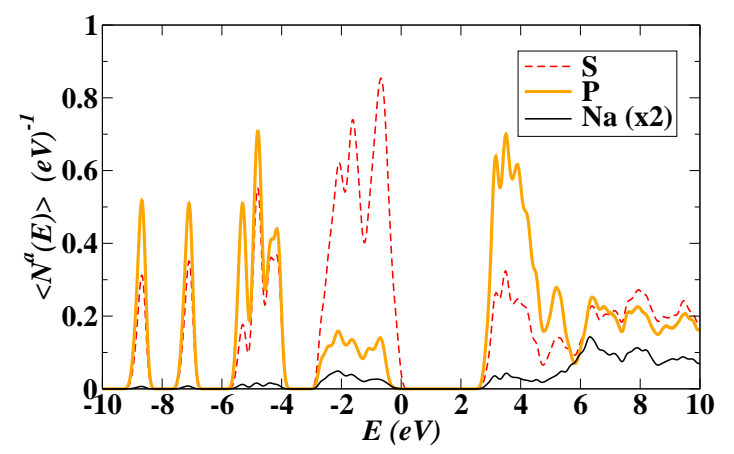

Figure 5: (Color online.) Partial density of states plot for $\mathrm{Na}_{4} \mathrm{P}_{2} \mathrm{~S}_{6}$ in the $C 2 / m$ structure. The contribution from $\mathrm{Na}$ has been scaled by a factor 2 in order increase its visibility.

In this case, the mappings are precise. As discussed in previous work[4] these two structures represent two of many of the ground state configurations of $\mathrm{Li}_{4} \mathrm{P}_{2} \mathrm{~S}_{6}$ and presumably of $\mathrm{Na}_{4} \mathrm{P}_{2} \mathrm{~S}_{6}$.

Table 3 lists the computed heats of formation per formula unit for $\mathrm{Na}_{4} \mathrm{P}_{2} \mathrm{~S}_{6}$ and $\mathrm{Li}_{4} \mathrm{P}_{2} \mathrm{~S}_{6}$ in each of the structures, which is referenced to the standard states of the elements given in the CRC Handbook[24]. The results show the Pnnm and Pnma structures, which represent two possible realizations of the disordered $P 6_{3} / \mathrm{mcm}$ structure of $\mathrm{Li}_{4} \mathrm{P}_{2} \mathrm{~S}_{6}$ analyzed by Mercier, [3] have the lowest energy for both $\mathrm{Na}_{4} \mathrm{P}_{2} \mathrm{~S}_{6}$ and $\mathrm{Li}_{4} \mathrm{P}_{2} \mathrm{~S}_{6}$. The $C 2 / m$ structure is $0.1 \mathrm{eV} /$ formula unit higher in energy for $\mathrm{Na}_{4} \mathrm{P}_{2} \mathrm{~S}_{6}$ and by $0.4 \mathrm{eV} /$ formula unit for $\mathrm{Li}_{4} \mathrm{P}_{2} \mathrm{~S}_{6}$. These results suggest that the synthesis method of Kuhn and co-workers[2] enabled them to produce this meta-stable phase of $\mathrm{Na}_{4} \mathrm{P}_{2} \mathrm{~S}_{6}$. The results also suggest that, because of its higher formation energy, it is unlikely that the analogous synthesis method would be successful for $\mathrm{Li}_{4} \mathrm{P}_{2} \mathrm{~S}_{6}$. The computational results also show that the $P \overline{3} 1 \mathrm{~m}$ structure is iso-energetic to the $C 2 / m$ structure for $\mathrm{Na}_{4} \mathrm{P}_{2} \mathrm{~S}_{6}$ and is $0.03 \mathrm{eV} /$ formula unit above the lowest energy structures for $\mathrm{Li}_{4} \mathrm{P}_{2} \mathrm{~S}_{6}$.

Table 3: Heats of formation (eV per formula unit) computed for the four optimized model structures labeled by their space groups and described in Tables 1 and 2.

\begin{tabular}{|c|c|c|}
\hline Structure & $\mathrm{Na}_{4} \mathrm{P}_{2} \mathrm{~S}_{6}$ & $\mathrm{Li}_{4} \mathrm{P}_{2} \mathrm{~S}_{6}$ \\
\hline$C 2 / m$ & -11.47 & -12.07 \\
\hline$P \overline{3} 1 m$ & -11.47 & -12.42 \\
\hline Pnnm & -11.56 & -12.46 \\
\hline Pnma & -11.56 & -12.46 \\
\hline
\end{tabular}

The partial densities of states for $\mathrm{Na}_{4} \mathrm{P}_{2} \mathrm{~S}_{6}$ is shown in Fig. 5 for the Kuhn structure; corresponding plots for the other three structures (not shown) are remarkably similar. The partial density of states for $\mathrm{Na}_{4} \mathrm{P}_{2} \mathrm{~S}_{6}$ is also very similar to that of $\mathrm{Li}_{4} \mathrm{P}_{2} \mathrm{~S}_{6}$ shown in Ref. [4], distinguished by the low energy phosphorus dimer states.
Table 4: Vacancy energies (in eV) for inequivalent sites as indicated by the Wyckoff site labels for three different crystal structures. In each case, the lowest energy site sets the zero of energy.

\begin{tabular}{|c|c|c|c|}
\hline Space Group & Site & $\mathrm{Na}_{4} \mathrm{P}_{2} \mathrm{~S}_{6}$ & $\mathrm{Li}_{4} \mathrm{P}_{2} \mathrm{~S}_{6}$ \\
\hline \multicolumn{4}{|l|}{$C 2 / m$; No. 12} \\
\hline & $\mathrm{Na} / \mathrm{Li} g$ & 0.40 & 0.52 \\
\hline & $\mathrm{Na} / \mathrm{Li} h$ & 0.00 & 0.00 \\
\hline \multicolumn{4}{|l|}{$P \overline{3} 1 m ;$ No. 162} \\
\hline & $\mathrm{Na} / \mathrm{Li} c$ & 0.35 & 0.32 \\
\hline & $\mathrm{Na} / \mathrm{Li} d$ & 0.00 & 0.00 \\
\hline \multicolumn{4}{|l|}{ Pnma; No. 62} \\
\hline & $\mathrm{Na} / \mathrm{Li} d$ & 0.15 & 0.11 \\
\hline & $\mathrm{Na} / \mathrm{Li} d$ & 0.00 & 0.00 \\
\hline
\end{tabular}

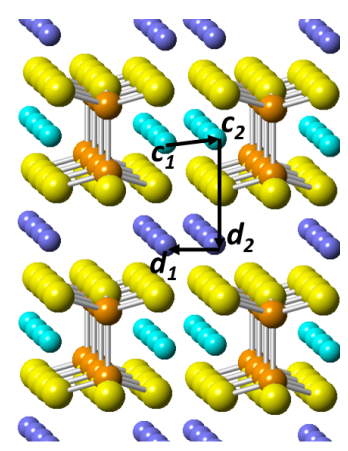

(a)

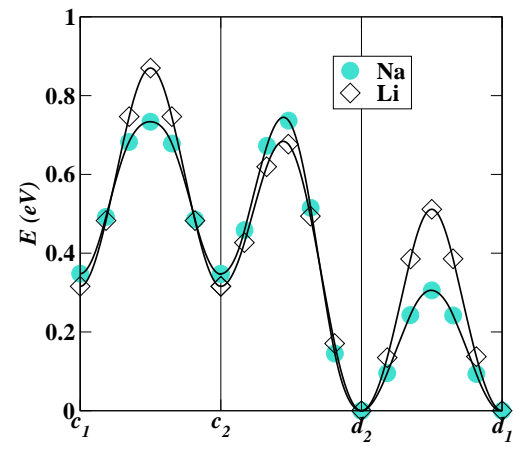

(b)
Figure 6: (Color online.) (a) Structural diagram for vacancy migration in $P \overline{3} 1 \mathrm{~m}$ structure of $\mathrm{Na}_{4} \mathrm{P}_{2} \mathrm{~S}_{6}$ viewed with the perspective as Fig. 1b. The Li ion vacancy sites are labeled according to the Wyckoff notation. (b) NEB diagram for $\mathrm{Na}$ or Li ion vacancy migration in the $P \overline{3} 1 \mathrm{~m}$ structure of $\mathrm{Na}_{4} \mathrm{P}_{2} \mathrm{~S}_{6}$ and $\mathrm{Li}_{4} \mathrm{P}_{2} \mathrm{~S}_{6}$.

\subsection{Ion migration}

We considered $\mathrm{Na} / \mathrm{Li}$ ion vacancy migration in three of the structures using supercells composed of 8 formula units. Table 4 lists the energies of each of the vacancy sites relative to the lowest energy site of each structure.

The $P \overline{3} 1 \mathrm{~m}$ structure results are given in Fig. 6 . The path including sites with Wyckoff label $c$ involves vacancy migration within planes perpendicular to the $c$-axis among the $\mathrm{P}_{2} \mathrm{~S}_{6}$ units while the path including sites with Wyckoff label $d$ involves vacancy migration within planes perpendicular to the $c$-axis between the $\mathrm{P}_{2} \mathrm{~S}_{6}$ units. The latter path has a lower overall energy with a barrier height of 0.5 and $0.3 \mathrm{eV}$ for $\mathrm{Li}$ and $\mathrm{Na}$, respectively. The migration barrier for the vacancy migrating between $d$ and $c$ sites is $E_{m}=0.7 \mathrm{eV}$ for both materials. Overall, the most favorable vacancy migration path for this material appears to be between $d$ sites in each hexagonal plane between $\mathrm{P}_{2} \mathrm{~S}_{6}$ units and the Na material has the lowest migration energy of $E_{m}=0.3 \mathrm{eV}$.

The Kuhn structure results are given in Fig. 7 where the similarities to the hexagonal structure results of Fig. 6 are evident where the sites with Wyckoff labels $g$ and $h$ for the $C 2 / m$ structure map to the sites with Wyckoff labels $c$ and $d$ in the $P \overline{3} 1 \mathrm{~m}$ structure, respectively. In- 


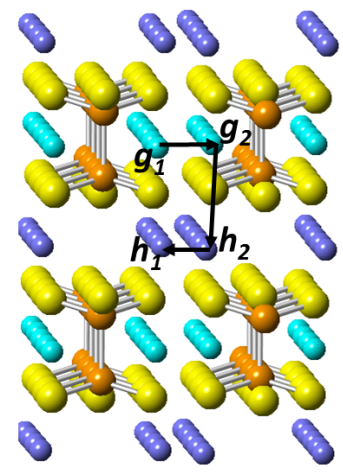

(a)

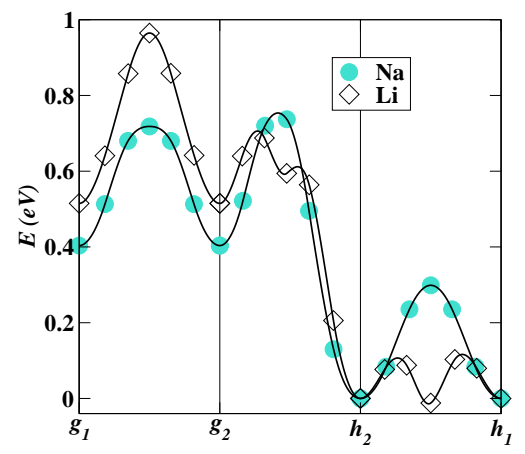

(b)

Figure 7: (Color online.) (a) Structural diagram for vacancy migration in $C 2 / m$ structure of $\mathrm{Na}_{4} \mathrm{P}_{2} \mathrm{~S}_{6}$ viewed from the same perspective as Fig. 2b. The Li ion vacancy sites are labeled according their Wyckoff notation. (b) NEB diagram for $\mathrm{Na}$ or $\mathrm{Li}$ ion vacancy migration in this structure of $\mathrm{Na}_{4} \mathrm{P}_{2} \mathrm{~S}_{6}$ and $\mathrm{Li}_{4} \mathrm{P}_{2} \mathrm{~S}_{6}$.

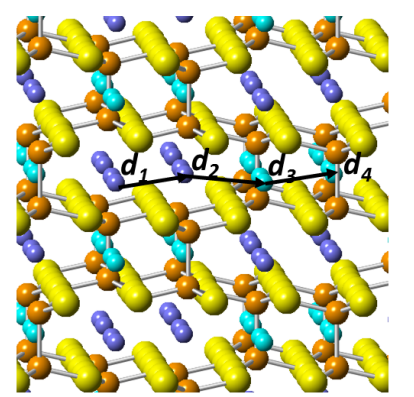

(a)

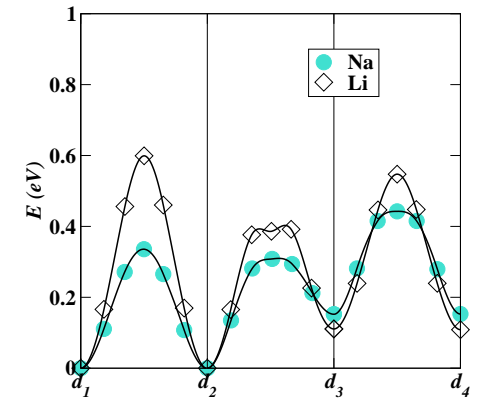

(b)

Figure 8: (Color online.) (a) Structural diagram for vacancy migration in Pnma structure of $\mathrm{Na}_{4} \mathrm{P}_{2} \mathrm{~S}_{6}$ with the vertical direction representing the $c$-axis and the horizontal direction representing the $b$-axis. Li ion vacancy sites are labelled according to their Wyckoff notation. (b) NEB diagram for $\mathrm{Na}$ or $\mathrm{Li}$ ion vacancy migration in the Pnma structure of $\mathrm{Na}_{4} \mathrm{P}_{2} \mathrm{~S}_{6}$ and $\mathrm{Li}_{4} \mathrm{P}_{2} \mathrm{~S}_{6}$.

terestingly, however, the $h_{1} \rightarrow h_{2}$ migration for the Li material in this structure has a very low energy barrier apparently due to a meta-stable interstitial site near the midpoint of the migration. These results indicate a very favorable Li ion vacancy migration path for this material if it can be made. For the $\mathrm{Na}$ material the $\mathrm{Na}$ ion vacancy migration barrier for this path is $E_{m}=0.3 \mathrm{eV}$. For this geometry, the most favorable path for $\mathrm{Na}$ ion vacancy migration consists of zigzag hops between $h$ sites with net motion along the a axis. For this geometry, we also estimated the minimum energy to form a vacancy-interstitial pair of $E_{f} \approx 0.2 \mathrm{eV}$, suggesting that the activation energy for $\mathrm{Na}$ ion diffusion should be $E_{A} \approx 0.4 \mathrm{eV}$. In this case, the meta-stable interstitial sites are located at the centers of the distored hexagonal pattern of the $h$ site $\mathrm{Na}$ ions. More complicated migration mechanisms involving active participation of the interstitial sites might be worth further investigation.

The vacancy migration for $\mathrm{Na}_{4} \mathrm{P}_{2} \mathrm{~S}_{6}$ and $\mathrm{Li}_{4} \mathrm{P}_{2} \mathrm{~S}_{6}$ in the Pnma structure, representing the lowest energy paths for

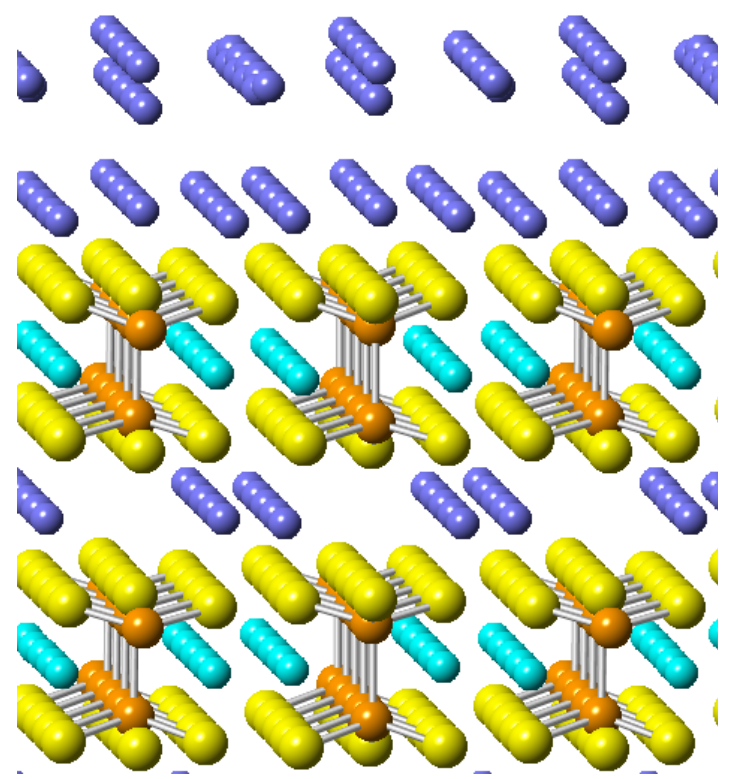

Figure 9: (Color online.) Ball and stick model of optimized metastable configuration of $\mathrm{Na} / \mathrm{Na}_{4} \mathrm{P}_{2} \mathrm{~S}_{6} / \mathrm{Na}$ interface for the Kuhn structure cleaved parallel to the pseudo-hexagonal plane using the same ball convention as in in Fig. 1.

these materials, is shown in Fig. 8. For this structure each $\mathrm{Na} / \mathrm{Li}$ has regions which are between $\mathrm{P}_{2} \mathrm{~S}_{6}$ units and other regions which span $\mathrm{P}_{2} \mathrm{~S}_{6}$ units and the migration energy barrier within a $\mathrm{Na} / \mathrm{Li}$ plane is generally smaller than the barrier to migrate between planes. The migration energies within a plane is shown in Fig. 8. The maximum energy barrier within this path is $E_{m}=0.6$ and $0.4 \mathrm{eV}$ for $\mathrm{Li}$ and $\mathrm{Na}$, respectively. For this structure, vacancy migration in the $c$-direction is generally energetically more costly than migration within a hexagonal plane.

\subsection{Surfaces and interfaces}

In our previous study of interfaces of $\mathrm{Li}_{4} \mathrm{P}_{2} \mathrm{~S}_{6}$ with lithium metal, both experimentally and computationally, we found the interface to be unstable. Experimentally, symmetric cells of $\mathrm{Li} / \mathrm{Li}_{4} \mathrm{P}_{2} \mathrm{~S}_{6} / \mathrm{Li}$ could be cycled only a few times. Computationally, optimized structures of $\mathrm{Li} / \mathrm{Li}_{4} \mathrm{P}_{2} \mathrm{~S}_{6} / \mathrm{Li}$ supercells resulted in a number of broken $\mathrm{P}-\mathrm{S}$ bonds and the formation $\mathrm{Li}_{2} \mathrm{~S}$ clusters at the interface. [4] Focusing on the Kuhn structure of $\mathrm{Na}_{4} \mathrm{P}_{2} \mathrm{~S}_{6}$, we computationally examined $\mathrm{Na} / \mathrm{Na}_{4} \mathrm{P}_{2} \mathrm{~S}_{6} / \mathrm{Na}$ supercells. We found that it is possible to generate idealized perfect interfaces such as shown in Fig. 9. The structure is very sensitive to the initial configuration and structures with broken $\mathrm{P}-\mathrm{S}$ bonds have lower energy, suggesting that the interface of $\mathrm{Na}_{4} \mathrm{P}_{2} \mathrm{~S}_{6}$ with sodium metal shown in Fig. 9 is marginally meta-stable, but perhaps slightly more stable than its $\mathrm{Li}$ analog. The partial density of states corresponding to the interface configuration of Fig. 9 is shown in Fig. 10. The Fermi level due to the metalic $\mathrm{Na}$ layers is aligned just below the unoccupied conduction bands of the electrolyte. 


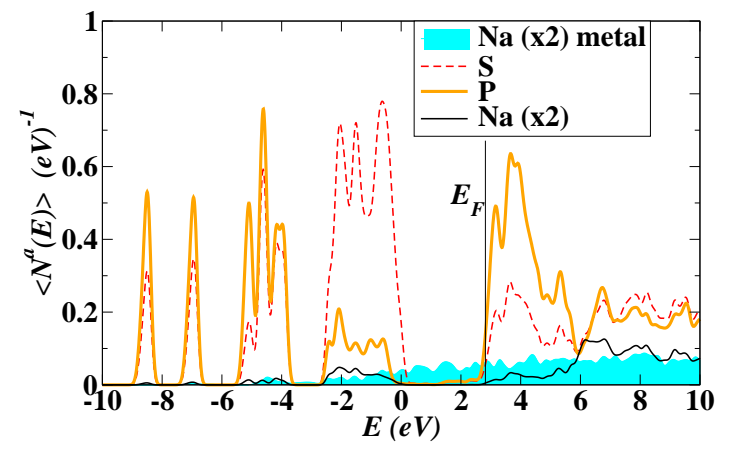

Figure 10: (Color online.) Partial density of states diagram of interface system shown in Fig. 9. The metallic Na sites are indicated separately from the sites explicitly associated with the electrolyte. Both the metallic and ionic $\mathrm{Na}$ sites are scaled by a factor of 2 for visibility.

\section{Discussions and conclusions}

The results of the current simulation study of $\mathrm{Na}_{4} \mathrm{P}_{2} \mathrm{~S}_{6}$ and the growing importance of solid electrolytes with favorable $\mathrm{Na}$ ion conductivity encourage further experimental investigation of this material. The challenges for further experimental study include the determination of factors which control the formation of the Kuhn structure versus the Mercier structure which the simulations find to be more stable by $0.1 \mathrm{eV}$ per formula unit. The simulations also suggest that the Kuhn structure, with its ordered placement of the $\mathrm{P}_{2} \mathrm{~S}_{6}$ building blocks, may have favorable ionic conductivity as indicated by the estimated activation energy of $E_{A}=0.4 \mathrm{eV}$. To our knowledge, the conductivity of $\mathrm{Na}_{4} \mathrm{P}_{2} \mathrm{~S}_{6}$ has not yet been measured.

\section{Acknowledgments}

This work was supported by NSF grants DMR-1105485 and DMR-1507942. Computations were performed on the Wake Forest University DEAC cluster, a centrally managed resource with support provided in part by the University.

\section{References}

[1] Akitoshi Hayashi, Kousuke Noi, Motohiro Tanibata, Naoto an d Nagao, and Masahiro Tatsumisago. High sodium ion conductivity of glassceramic electrolytes with cubic $\mathrm{Na}_{3} \mathrm{PS}_{4}$. Journal of Power Sources, 258:420-423, 2014.

[2] Alexander Kuhn, Roland Eger, Jürgen Nuss, and Bettina V. Lotsch. Synthesis and structural characterization of the alkali thiophosphates $\mathrm{Na}_{2} \mathrm{P}_{2} \mathrm{~S}_{6}, \mathrm{Na}_{4} \mathrm{P}_{2} \mathrm{~S}_{6}, \mathrm{~K}_{4} \mathrm{P}_{2} \mathrm{~S}_{6}$, and $\mathrm{Rb}_{4} \mathrm{P}_{2} \mathrm{~S}_{6}$. Zeitschrift für anorganische und allgemeine Chemie, 640(5):689-692, 2014.

[3] R. Mercier, J. P. Malugani, B. Fahys, J. Douglade, and G. Robert. Synthese, structure cristalline at analyse vibrationnelle de l'hexathiohypodiphosphate de lithium $\operatorname{Li}_{4} \mathrm{P}_{2} \mathrm{~S}_{6}$. Journal of Solid State Chemistry, 43:151-162, 1982.

[4] Zachary D. Hood, Cameron Kates, Melanie Kirkham, Shiba Adhikari, Chengdu Liang, and N. A. W. Holzwarth, 2015. Submitted for publication.
[5] N. D. Lepley, N. A. W. Holzwarth, and Yaojun A. Du. Structures, Li+ mobilities, and interfacial properties of solid electrolytes $\mathrm{Li}_{3} \mathrm{PS}_{4}$ and $\mathrm{Li}_{3} \mathrm{PO}_{4}$ from first principles. Phys. Rev. $B, 88: 104103$ (11 pp), 2013.

[6] P. Hohenberg and W. Kohn. Inhomogeneous electron gas. Physical Review, 136:B864-B871, 1964.

[7] W. Kohn and L. J. Sham. Self-consistent equations including exchange and correlation effects. Physical Review, 140:A1133A1138, 1965.

[8] P. E. Blöchl. Projector augmented-wave method. Phys. Rev. B, 50:17953-17979, 1994.

[9] N. A. W. Holzwarth, G. E. Matthews, R. B. Dunning, A. R. Tackett, and Y. Zeng. Comparison of the projector augmented wave, pseudopotential, and linearized augmented plane wave formalisms for density functional calculations of solids. Phys. Rev. B, 55:2005-2017, 1997.

[10] N. A. W. Holzwarth, A. R. Tackett, and G. E. Matthews. A Projector Augmented Wave (PAW) code for electronic structure calculations, Part I: atompaw for generating atom-centered functions. Computer Physics Communications, 135:329-347, 2001. Available from the website http://pwpaw.wfu.edu.

[11] X. Gonze, B. Amadon, P. M. Anglade, J. M. Beuken, F. Bottin, P. Boulanger, F. Bruneval, D. Caliste, R. Caracas, M. Cote, T. Deutsch, L. Genovese, Ph. Ghosez, M. Giantomassi, S. Goedecker, D. R. Hamann, P. Hermet, F. Jollet, G. Jomard, S. Leroux, M. Mancini, S. Mazevet, M. J. T. Oliveira, G. Onida, Y. Pouillon, T. Rangel, G. M. Rignanese, D. Sangalli, R. Shaltaf, M. Torrent, M. J. Verstraete, G. Zerah, and J. W. Zwanziger. Abinit: First-principles approach to material and nanosystem properties. Computer Physics Communications, 180(12):2582-2615, 2009. Code is available at the website http: //www . abinit.org.

[12] Paolo Giannozzi, Stefano Baroni, Nicola Bonini, Matteo Calandra, Roberto Car, Carlo Cavazzoni, Davide Ceresoli, Guido L Chiarotti, Matteo Cococcioni, Ismaila Dabo, Andrea Dal Corso, Stefano de Gironcoli, Stefano Fabris, Guido Fratesi, Ralph Gebauer, Uwe Gerstmann, Christos Gougoussis, Anton Kokalj, Michele Lazzeri, Layla Martin-Samos, Nicola Marzari, Francesco Mauri, Riccardo Mazzarello, Stefano Paolini, Alfredo Pasquarello, Lorenzo Paulatto, Carlo Sbraccia, Sandro Scandolo, Gabriele Sclauzero, Ari P Seitsonen, Alexander Smogunov, Paolo Umari, and Renata M Wentzcovitch. Quantum espresso: a modular and open-source software project for quantum simulations of materials. J. Phys.: Condens. Matter, 21(39):394402 (19pp), 2009. Available from the website http://www.quantum-espresso.org.

[13] John P. Perdew and Yue Wang. Accurate and simple analytic representation of the electron-gas correlation energy. Phys. Rev. $B, 45: 13244-13249,1992$.

[14] H. T. Stokes and D. M. Hatch. Findsym: Program for identifying the space group symmetry of a crystal. J. Appl. Cryst., 38:237-238, 2008. Available from the webpage http://iso.byu. edu/iso/isotropy.php.

[15] H. Jónsson, G. Mills, and K. W. Jacobsen. Nudged elastic band method for finding minimum energy paths of transitions. In B. J. Berne, G. Ciccotti, and D. F. Coker, editors, Classical and Quantum Dynamics in Condensed Phase Simulations, pages 385-404. World Scientific, Singapore, 1998.

[16] Graeme Henkelman, Blas P. Uberuaga, and Hannes Jónsson. A climbing image nudged elastic band method for finding saddle points and minimum energy paths. J. Chem. Phys., 113:99019904, 2000.

[17] Graeme Henkelman and Hannes Jónsson. Improved tangent estimate in the nudged elastic band method for finding minimum energy paths and saddle points. J. Chem. Phys., 113:9978-9985, 2000 .

[18] Anthony R. West. Basic Solid State Chemistry, second edition. John Wiley \& Sons, LTD, 1999.

[19] Anton Kokalj. XCrySDen- an new program for displaying crystalline structures and densities. Journal of Molecular Graphics and Modelling, 17:176-179, 1999. Code available at the website 
http://www . xcrysden.org.

[20] Anton Kokalj. Computer graphics and graphical user interfaces as tools in simulations of matter at the atomic scale. Computational Materials Science, 28:155-168, 2003.

[21] Koichi Momma and Fujio Izumi. Vesta 3 for three-dimensional visualization of crystal, volumetric, an morphology data. $A p$ plied Crystallography, 44:1272-1276, 2011. Code available from the website http://jp-minerals.org/vesta/en/.

[22] Crystalmaker, 2015. Propriatory software available from the website www.crystalmaker.com.

[23] Th. Hahn, editor. International Tables for Crystallography, Volume A: Space-group symmetry, Fifth revised edition. Kluwer, 2002. ISBN 0-7923-6590-9.

[24] W. M. Haynes, editor. CRC Handbook of Chemistry and Physics, 92th Edition. CRC Press, Taylor \& Francis Group, 2011. ISBN 978-1-4398-5511-9. 